\title{
Prostate Carcinoma
}

National Cancer Institute

\section{Source}

National Cancer Institute. Prostate Carcinoma. NCI Thesaurus. Code C4863.

One of the most common malignant tumors afflicting men. The majority of carcinomas arise in the peripheral zone and a minority occur in the central or the transitional zone of the prostate gland. Grossly, prostatic carcinomas appear as ill-defined yellow areas of discoloration in the prostate gland lobes. Adenocarcinomas represent the overwhelming majority of prostatic carcinomas. Prostatic-specific antigen (PSA) serum test is widely used as a screening test for the early detection of prostatic carcinoma. Treatment options include radical prostatectomy, radiation therapy, androg en ablation and cryotherapy. Watchful waiting or surveillance alone is an option for older patients with low-grade or low-stage disease. -- 2002 